\title{
ESTUDO DE ADAPTAÇÃO E VALIDAÇÃO DE UM QUESTIONÁRIO DE CLIMA ORGANIZACIONAL PARA ORGANIZAÇÕES SOCIAIS
}

\author{
Margarida V. Garrido ${ }^{1}$ \\ Diniz Lopes ${ }^{1}$ \\ Maria Manuela Calheiros ${ }^{1}$
}

Resumo: O presente estudo tem como objectivo adaptar e validar um questionário de clima organizacional para instituições sociais, a partir do Organizational Climate Survey (The University of Tennessee Children's Mental Health Services Research Center, 2000), utilizando uma amostra de profissionais que trabalham em diversas áreas de intervenção social. O instrumento original apresenta uma abordagem multidimensional do conceito de clima, integrando uma estrutura com quatro temas gerais (clima, estrutura, tecnologia e atitudes face ao trabalho). Após a tradução do instrumento, e de forma a observar as qualidades psicométricas da versão portuguesa, procedeu-se à análise da sua estrutura factorial através de análises factoriais confirmatórias, análise da fidelidade, análise da sensibilidade e validação relativa a um critério. Os resultados da análise factorial confirmatória indicaram que o modelo original é suportado em apenas três dos quatro factores do questionário original. As análises de fidelidade realizadas revelam uma consistência interna adequada de cada factor, e as análises de sensibilidade sugerem que o instrumento é sensível a diferenças individuais. Os resultados da validação relativa a um critério (i.e., validade concorrente) mostraram, ainda, evidência preliminar de que as dimensões de clima organizacional se associam às percepções de satisfação e implicação organizacional dos inquiridos.

Palavras-Chave: clima organizacional, organizações sociais, validação, sensibilidade

Adaptation and validation of a questionnaire of organizational climate for social welfare organizations (Abstract): The present study aims at adapting and validating an organizational climate questionnaire for social organizations, based on the Organizational Climate Survey (The University of Tennessee: Children's Mental Health Services Research Center, 2000), using a sample of professionals working in several social welfare organizations. The original instrument entails a multidi-

${ }^{1}$ CIS / ISCTE - Instituto Universitário de Lisboa 
mensional approach to the concept of climate and is characterized by a structure with four general themes (climate, structure, technology and work attitudes). After translating the instrument and in order to explore the psychometric characteristics of the Portuguese version we analyzed the factorial structure through confirmatory factorial analyses, reliability, sensitivity and criterion-related validity. Results from confirmatory factorial analysis indicate that the original model is supported in only three of the four factors of the original questionnaire. Reliability analyses showed adequate levels of internal consistency for each factor and sensitivity analysis suggested that the instrument is sensitive to individual differences. Criterion-related validation results (i.e., concurrent validity) showed preliminary evidence for the association of the dimensions of organizational climate with respondents' perceptions of satisfaction and organizational commitment.

Keywords: organizational climate, social welfare organizations, validation, sensitivity

\section{Introdução}

O contexto social de uma organização inclui um conjunto de factores psicossociais, nomeadamente relações interpessoais, normas sociais, expectativas comportamentais, e atitudes que determinam, entre outros aspectos, a abordagem ao trabalho, os sentimentos face ao papel organizacional, a interacção intra e inter-organizacional e a interpretação do ambiente laboral por parte dos colaboradores (Glisson, 2002).

É neste contexto que os estudos de clima organizacional procuram, através da percepção dos colaboradores, explorar a realidade organizacional e identificar um conjunto de aspectos que afectam o seu comportamento, atitudes, bem-estar, desempenho profissional e, consequentemente, os determinantes do sucesso organizacional (Glisson \& James, 2002).

Com base nesta informação é possível gerir o clima e planear intervenções organizacionais que maximizem a qualidade de vida no trabalho, o desempenho e a produtividade organizacional, tal como documentado por um amplo conjunto de evidência empírica (e.g., Kleinman, Handal, Enos, Searight, \& Ross, 1989; Kottkamp, Mulhern, \& Hoy, 1987; O’Driscoll \& Evans, 1988; Poole, 1985; Turnipseed, 1980; Witt, 1989). A monitorização do clima organizacional constitui, assim, uma importante ferramenta de gestão na medida em que veicula informação, promove a participação dos colaboradores na expressão da sua visão organizacional, favorece a comunicação intra-organizacional, permite comparar diferentes percepções dentro de uma mesma ou de diferentes organizações, promove e reforça boas-práticas e aumenta o envolvimento dos colaboradores no trabalho, com repercussões nas taxas de absentismo, satisfação e desempenho (e.g., Altmann, 2000). 
Atendendo à relevância da avaliação e monitorização do clima organizacional, o objectivo do presente artigo é, então, o de apresentar um estudo de adaptação e validação de um instrumento de medida de clima organizacional específico para organizações sociais.

\section{Clima Organizacional}

O clima organizacional é um construto complexo e multidetermidado e, como tal, susceptível de muitas definições, sendo talvez a mais consensual aquela que o define como o modo através do qual as pessoas percebem os seus ambientes de trabalho (e.g., James \& James, 1989; Verbeke, Volgering, \& Hessels, 1998).

O conceito de clima organizacional tem sido abordado sob quatro perspectivas distintas: organizacional, psicológica, psicossocial e cultural (Neves, 2001). A perspectiva organizacional, onde o clima é encarado como uma manifestação objectiva das características da organização, reflectindo o conjunto de percepções semelhantes que emergem da exposição dos mesmos indivíduos aos mesmos factores organizacionais (e.g., Angle \& Perry, 1986; De Witte \& De Cock, 1986; Forehand \& Gilmer, 1964; Guion, 1973; Miceli \& Near, 1985; Payne \& Pugh, 1975); a perspectiva psicológica, no âmbito da qual o clima expressa a forma como o indivíduo, mediado pelas suas características individuais, percebe a realidade organizacional (e.g., James, James, \& Ashe, 1990; James \& Jones, 1974; Joyce \& Slocum, 1982, 1984); a perspectiva psicossocial, segundo a qual a interpretação da realidade organizacional decorre da percepção das características organizacionais, da sua interacção com outros membros da organização e respectivas relações de poder e ainda de processos políticos (Mintzberg, 1979), processos de pertença grupal (Newman, 1977), e processos simbólicos de interacção (Festinger, 1954; Schneider \& Reichers, 1983); finalmente, a perspectiva cultural centra-se na influência de um contexto mais amplo, onde um grupo de indivíduos interage e partilha uma estrutura de referência que contextualiza a sua interacção e o significado dos eventos organizacionais (a cultura organizacional; Moran \& Volkwein, 1992).

\section{Clima em organizações sociais}

O impacto do clima organizacional no desempenho dos profissionais e das próprias organizações tem-se constituído como foco de extensa pesquisa desde a década de 70 (e.g., Hellriegel \& Slocum, 1974; James \& Jones, 1974; Payne \& Pugh, 1975; Schneider, 1975). Contudo, esta pesquisa tem-se centrado sobretudo em organizações industriais e empresariais. De facto, apesar de os resultados indicarem que o clima organizacional constitui um factor relevante na qualidade dos serviços prestados por organizações de 
outra natureza, o estudo sistemático do clima só muito lentamente se tem vindo a generalizar a instituições na área educativa (Ostroff \& Schmitt, 1993; Soloman, 1986) e sócio-educativa (Glisson \& Hemmelgarn, 1998; Shim, 2010; Yoo, Brooks, \& Patti, 2007), saúde (Carvalho, 2007; Paulin, Ferguson, \& Bergeron, 2006), justiça e saúde mental (Glisson \& James, 2002).

No contexto das organizações de saúde, alguns estudos sugerem que um clima organizacional positivo se relaciona com a saúde e o bem-estar dos profissionais de saúde (e.g., Carvalho, 2007), promove a satisfação e o compromisso dos profissionais com a organização, e fomenta comportamentos conducentes à percepção da satisfação do cliente e da qualidade do serviço prestado (e.g., Paulin et al., 2006). O clima mostrou-se também um bom preditor da rotatividade organizacional, tal como indicam os trabalhos de Shim (2010) realizados no contexto de serviços sociais de apoio à infância, destacando a exaustão emocional como um dos factores mais significativos na explicação das intenções dos colaboradores para abandonarem a organização. Também no âmbito do sistema de protecção de crianças e jovens em risco, Glisson e Hemmelgarn (1998) mostram a existência de uma relação entre o clima organizacional (especificamente no que toca ao baixo conflito, à cooperação, à satisfação laboral e à personalização) e a qualidade do serviço prestado.

A particular relevância que o clima organizacional assume nas organizações sociais baseia-se, sobretudo, nos objectivos destas organizações e na natureza do trabalho desenvolvido pelos seus colaboradores. Trata-se assim de organizações que prestam serviços continuados a populações vulneráveis, frequentemente em risco de uma variedade de problemas físicos, sociais e psicológicos. Estes aspectos tornam o trabalho nestas organizações imprevisível, complexo e sujeito a elevados níveis de stresse, aliado a uma constante necessidade de lidar com uma teia burocrática complexa de regulamentos legais e organizacionais. Acresce que, aliado às fortes exigências de tempo e de energia, estas funções são frequentemente acompanhadas por baixas remunerações (Glisson \& James, 2002). Por outro lado, a eficácia dos serviços de intervenção social e o sucesso dos seus resultados dependem fortemente das inter-relações laborais (baseadas muitas vezes na confiança), de um ambiente de trabalho positivo e de uma força de trabalho competente e estável, capaz de dar resposta a problemas inesperados e a necessidades individualizadas das populações que servem (Glisson, Dukes, \& Green, 2006).

No entanto, a investigação empírica tem demonstrado que muitas destas organizações se caracterizam por atitudes laborais negativas (Glisson \& Durick, 1988), elevadas taxas de rotatividade e baixa satisfação com o trabalho, quando comparadas com outro tipo de organizações (Soloman, 1986), e que elevados níveis de stress e de conflito, e situações de burnout experien- 
ciadas por muitos trabalhadores, se encontram entre as variáveis que caracterizam o clima dos seus ambientes de trabalho. Indicadores de clima, tais como conflito de papel, exaustão emocional e despersonalização, também presentes nestas organizações, afectam quer a qualidade, quer os resultados do serviço prestado (Glisson \& Hemmelgarn, 1998), e explicam ainda as elevadas taxas de rotatividade observadas nas organizações que prestam serviços na área social (Cyphers, 2001).

Apesar deste cenário relativamente negativo, alguns estudos realizados em organizações sociais mostram que é possível intervir com sucesso nestas organizações através de estratégias de mudança organizacional (Glisson et al., 2006). Estes estudos apontam a promoção de climas organizacionais positivos como um dos principais preditores de atitudes de trabalho positivas, de menores taxas de rotatividade, dum maior esforço para ultrapassar obstáculos, do aumento da resposta às necessidades dos utentes e da promoção de serviços de elevada qualidade (Glisson \& Durick 1988; Glisson \& Hemmelgarn, 1998, Glisson \& James, 2002; Henggeler \& Schoenwald, 1999).

\section{Objectivos}

No presente artigo, apresentamos a adaptação e validação de um questionário de clima organizacional, desenvolvido para aplicação em organizações sociais, a uma amostra portuguesa de colaboradores deste tipo de organizações. O questionário em causa foi elaborado pelo Children's Mental Health Services Research Center da University do Tennessee, EUA, e baseia-se no questionário de clima psicológico de James e Sells (1981), adaptado por Glisson e Hemmelgarn (1998). Para tal, analisámos as suas qualidades psicométricas, nomeadamente através da análise da estrutura factorial e do teste à fidelidade e sensibilidade. Os dados obtidos com este questionário foram, também, explorados em função de algumas variáveis sociodemográficas, nomeadamente, idade, sexo, cargo de chefia e função ocupada pelos inquiridos (análises de sensibilidade do instrumento). Além disto, explorámos ainda como as percepções do clima organizacional podem afectar outras variáveis importantes do contexto de trabalho, tais como a satisfação e implicação organizacionais, explorando a validade relativa a um critério (validade concorrente) deste mesmo instrumento.

Do ponto de vista teórico, o estudo do clima em instituições sociais poderá constituir um contributo importante para a validação e generalização do conceito de clima e para a análise das suas implicações na eficácia e resultados organizacionais nestes contextos. Do ponto de vista da intervenção social, poderá revelar-se um contributo importante para o conhecimento deste tipo de instituições, e para a consequente melhoria da qualidade da prestação dos serviços às comunidades que servem. 


\section{Método}

\section{Amostra}

A amostra deste estudo é composta por 682 participantes, colaboradores de Organizações Sociais, tendo os dados sido recolhidos entre 2007 e $2009^{2}$. A média de idades dos participantes é de 37.11 anos $(D P=10.34)$, sendo $82.8 \%$ do sexo feminino. Cerca de metade dos participantes possui uma licenciatura $(50 \%)$, e $9.4 \%$ possui uma pós-graduação. De uma forma geral, estes participantes encontram-se a trabalhar na sua organização há cerca de oito anos, e há cerca de 10 anos em organizações desta natureza, sendo que $12.4 \%$ ocupa um lugar de chefia. Parte dos inquiridos (42.4\%) trabalha em organizações particulares de intervenção social na comunidade (IPSS) e cerca de 30\% em organizações governamentais de intervenção social na comunidade (e.g., Santa Casa da Misericórdia, Casa Pia de Lisboa, Autarquias, etc.). Os restantes inquiridos distribuem-se entre organizações de educação e formação $(22.6 \%)$ e de saúde (5.1\%). Quanto às funções ocupadas, verifica-se alguma heterogeneidade salientando-se, contudo, percentagens razoáveis de técnicos ${ }^{3}(12.7 \%)$, de profissionais de serviço social $(9.2 \%)$, de educadores $(16.8 \%)$, e de formadores/professores $(10.3 \%)$, e ainda um elevado número de profissionais com funções de auxiliar (17.9\%).

\section{O Questionário de Clima Organizacional em Instituições Sociais}

O questionário de clima organizacional utilizado neste estudo - o Organizational Climate Survey (OCL), desenvolvido pelo Children's Mental Health Services Research Center da University do Tennessee, EUA - baseia-se no questionário de clima psicológico de James e Sells (1981), adaptado por Glisson e Hemmelgarn (1998) para ser aplicado em instituições sociais de apoio à infância. $\mathrm{O}$ questionário de James e Sells tem por base o modelo de clima psicológico de Jones e James (1979) e mede a forma como os indivíduos percebem o seu ambiente de trabalho, e como utilizam esta sua percepção para avaliar as características do mesmo. Assim, o questionário utilizado por Glisson e Hemmelgarn retoma, não só, algumas dimensões centrais

\footnotetext{
${ }^{2}$ Os dados utilizados neste artigo resultam da aplicação de questionários por alunos da Unidade Curricular de Métodos de Investigação em Psicologia do Mestrado de Psicologia Comunitária e Protecção de Menores do ISCTE - Instituto Universitário de Lisboa a quem deixamos o nosso agradecimento.

3 Os inquiridos aqui designados como "técnicos" são profissionais licenciados na sua maioria em outras áreas das ciências sociais (e.g., sociologia, economia, etc.) que exercem funções na carreira técnica destas organizações sociais.
} 
do modelo de Jones e James (1979), tais como a clareza, o conflito e a sobrecarga de papéis, a cooperação ou a formalização e a hierarquia, como acrescenta outras dimensões adaptadas do questionário de James e Sells, tais como a despersonalização, a exaustão emocional, a justiça, o desenvolvimento pessoal, etc. Assim, o questionário original de Glisson e Hemmelgarn encontra-se dividido em quatro temas gerais - um tema de clima organizacional, e três temas de caracterização genérica de instituições e dos seus colaboradores: estrutura, tecnologia e atitudes face ao trabalho -, subdividindo cada tema em dimensões específicas. Este questionário possui, portanto, 14 dimensões e um total de 115 itens. De acordo com estudos anteriores (Glisson \& James, 2002), estas dimensões apresentam índices de consistência interna aceitáveis - clima: despersonalização ( $\alpha=.69 ; 5$ itens); exaustão emocional ( $\alpha=.92 ; 6$ itens); justiça ( $\alpha=.68 ; 6$ itens); crescimento e desenvolvimento $(\alpha=.80 ; 5$ itens); realização pessoal ( $\alpha=.72 ; 6$ itens); clareza de papéis $(\alpha=.83 ; 6$ itens); conflito de papéis ( $\alpha=.87 ; 9$ itens); sobrecarga de papéis $(\alpha=.85 ; 8$ itens); cooperação ( $\alpha=.81 ; 4$ itens); estrutura: formalização $(\alpha=.74 ; 8$ itens); hierarquia de autoridade ( $\alpha=.78 ; 7$ itens $)$; tecnologia: rotinização $(\alpha=.77 ; 5$ itens); atitudes face ao trabalho: satisfação no trabalho $(\alpha=.85 ; 11$ itens); implicação organizacional ( $\alpha=.88 ; 13$ itens).

De seguida, descrevemos os temas e dimensões deste instrumento ${ }^{4}$, fornecendo exemplos dos itens que as integram. A versão integral do questionário, adaptado e validado para a presente amostra, é apresentada no Anexo I.

\section{Tema-Clima}

O tema Clima é composto por nove dimensões: a dimensão despersonalização mede sentimentos de impessoalidade do técnico na relação com os utentes/clientes (e.g., "Sinto que trato alguns utentes/clientes de forma impessoal"; "É difícil sentir-me próximo(a) dos meus utentes/clientes"); a dimensão exaustão emocional mede sentimentos de fadiga e esgotamento relativamente à actividade exercida (e.g., "Sinto-me emocionalmente esgotado(a) com o meu trabalho"; "Sinto que emocionalmente estou a chegar ao meu limite"); a dimensão justiça é medida nas suas dimensões de justiça distributiva, processual e relacional (e.g., "Comparando-me com pessoas de outras organizações, considero que o meu ordenado é justo"; "Aqui as promoções são determinadas pelas políticas da organização"; "Aqui, ser promovido(a) depende mais do facto de gostarem de nós do que de fazer um bom

4 O tema "tecnologia", mais especificamente a sua dimensão "rotinização", não será alvo de análise no presente estudo uma vez que mostrou um nível de consistência interna muito baixo $(\alpha=.59)$ na presente amostra. 
trabalho"); a dimensão crescimento e desenvolvimento procura aferir a percepção dos indivíduos relativamente às suas possibilidades de promoção e avanço na carreira, bem como do seu próprio desenvolvimento profissional (e.g., "Esta organização enfatiza o crescimento e o desenvolvimento"; "Esta organização proporciona várias oportunidades de promoção se trabalharmos para tal"); na dimensão realização pessoal é avaliada a realização percebida pelos indivíduos no exercício da sua função ("Sinto-me bem-disposto(a) depois de trabalhar de perto com os meus utentes/clientes"; "Neste trabalho tenho realizado muitas coisas que valem a pena"); nas dimensões clareza, conflito e sobrecarga de papéis, são avaliadas as percepções dos indivíduos relativamente a aspectos processuais das instituições onde se encontram a trabalhar, nomeadamente à forma como o trabalho é organizado e conduzido dentro da organização (e.g., "Com que frequência os objectivos e propósitos do seu cargo estão claramente definidos?"), ao conflito entre a situação de trabalho dentro da instituição e a vida pessoal do colaborador (e.g., "Com que frequência o seu trabalho interfere com a sua vida familiar?), e à sobrecarga de trabalho percebida pelo indivíduo (e.g., "Por mais que eu faça, há sempre mais a ser feito"); finalmente, a dimensão cooperação diz respeito à forma como os indivíduos percebem as relações que estabelecem com os colegas da instituição (e.g., "Quando tenho uma tarefa difícil a fazer, as pessoas da organização ajudam-me"; "Com que frequência os seus colegas confiam uns nos outros?").

\section{Tema-Estrutura}

Os aspectos relativos à Estrutura Organizacional centram-se nas questões de centralização de poder e da formalização dos papéis dentro da organização (Carvalho, 2007) e incluem dados sobre a participação na tomada de decisão, sobre a hierarquia de autoridade, a divisão do trabalho e especificações a nível procedimental que guiam as relações de trabalho de membros de uma organização. No presente questionário, duas dimensões da estrutura organizacional são medidas: formalização e hierarquia de autoridade. A dimensão formalização centra-se na percepção dos indivíduos relativamente a procedimentos de trabalho na organização (e.g., "Aqui, as pessoas fazem o trabalho da mesma forma todos os dias"; "Devemos sempre seguir procedimentos operacionais rígidos"). A dimensão hierarquia de autoridade permite aceder à forma como os indivíduos percebem a hierarquia existente na sua organização e o estilo de tomada de decisão (e.g., "Poucas coisas podem ser feitas antes que um supervisor ou coordenador aprove a decisão"; "Todas as decisões que tomo têm que ser aprovadas pelo supervisor ou coordenador"). 


\section{Tema-Atitudes face ao Trabalho}

A investigação no campo das Atitudes face ao Trabalho inclui, normalmente, a análise da satisfação face ao trabalho e da implicação organizacional (Glisson \& Durick, 1988). A satisfação face ao trabalho é definida como uma avaliação positiva do trabalho ou das experiências com ele relacionadas (Locke, 1976), e diz respeito aos aspectos mais práticos do trabalho, isto é, às obrigações e tarefas dos indivíduos enquanto colaboradores de uma organização (Mowday, Porter, \& Steers, 1982; Williams \& Hazer, 1986). A implicação organizacional aparece definida na literatura enquanto uma motivação para permanecer membro de uma organização e de exercer um esforço adequado para atingir os objectivos por ela fixados (Mowday et al., 1982), sendo muitas vezes vista como uma forma de vinculação do indivíduo à organização.

A dimensão satisfação com o trabalho é operacionalizada através de itens como: "Com que frequência se sente satisfeito(a) com o prestígio que o seu trabalho tem na comunidade?"; "Com que frequência se sente satisfeito(a) com o reconhecimento que recebe por fazer um bom trabalho?" A dimensão implicação organizacional é operacionalizada através de itens como: "Sinto orgulho em dizer aos outros que faço parte desta organização"; "Estou disposto(a) a empenhar-me para que esta organização tenha sucesso".

\section{Procedimento}

No presente estudo, os participantes responderam a uma versão traduzida para português deste questionário. A tradução dos itens de inglês para português foi revista por um painel de três juízes, tendo sido seguida de retroversão por um tradutor profissional e nova avaliação pelo mesmo painel (Calheiros, Moleiro, Garrido, Rodrigues, \& Martins, 2010). Os profissionais que aceitaram participar no preenchimento deste questionário avaliaram cada item de acordo com uma escala de 5 pontos, variando entre $5=$ Sempre a $1=$ Nunca. No final, os participantes responderam, também, a algumas questões de caracterização sociodemográfica, nomeadamente, sexo, idade, habilitações, área de formação, organização de pertença, anos na área de trabalho, anos na organização, e exercício de função de chefia. O tempo máximo de preenchimento deste questionário ronda os 45 minutos. 


\section{Resultados}

Validade de Construto e análise da Consistência Interna do Questionário de Clima Organizacional

Para a determinação da validade de construto das dimensões que compõem os temas Clima, Estrutura e Atitudes face ao Trabalho deste questionário, realizámos diversas análises factoriais confirmatórias seguindo o modelo teórico e as estruturas factoriais propostas por Glisson e Hemmelgarn (1998).

Assim, para o tema Clima testámos um modelo confirmatório com as nove dimensões correlacionadas entre si que descrevemos anteriormente. Este modelo revelou que os itens que compõem a dimensão justiça não saturam significativamente no factor latente previsto no modelo. Por este facto, o modelo revelou-se muito inadequado. Assim, testámos um novo modelo com oito dimensões correlacionadas. Paralelamente, testámos um modelo com estas mesmas oito dimensões, desta vez não correlacionadas. Finalmente, foi testado um modelo factorial de segunda ordem.

Relativamente ao tema Estrutura foram calculados, novamente, três modelos e verificados os respectivos índices de adequação: um modelo a dois factores não correlacionados, um modelo a dois factores correlacionados, e um modelo de segunda ordem, ou seja, dois factores de primeira ordem associados a um factor latente de segunda ordem.

Por fim, e no que toca ao tema Atitudes face ao Trabalho foram, novamente, calculados três modelos: um modelo a dois factores correlacionados, um modelo a dois factores não correlacionados, e um modelo de segunda ordem.

No cálculo destes diferentes modelos confirmatórios foram aplicados os constrangimentos usuais para a obtenção da especificação geral e identificação destes modelos (cf. Arbuckle, 2006; Byrne, 2009), isto é, em cada factor latente o caminho entre um item e esse factor foi constrangido a $1 \mathrm{e}$ todos os erros de medida associados aos diferentes itens foram, também, constrangidos a 1. Igualmente, e por questões de identificação, no modelo de segunda ordem as variâncias únicas associadas aos factores de primeira ordem foram todas constrangidas à igualdade (cf., Byrne, 2009). Obtiveram-se índices de adequação relativos e absolutos dos modelos, ou seja, os índices qui-quadrado $\left(\chi^{2}\right)$, qui-quadrado relativo $\left(\chi^{2} / \mathrm{df}\right)$, o goodness-of-fit-index (GFI; Jöreskog \& Sorbom, 1984), o comparative-fit-index (CFI; Bentler, 1990), e o root-mean-square-error-of-approximation (RMSEA; Browne \& Cudeck, 1993). Os resultados destas diferentes análises factoriais confirmatórias são apresentados no Quadro 1. 
Quadro 1. Resumo dos índices de adequação dos modelos de análise factorial confirmatória realizados para os temas Clima, Estrutura e Atitudes face ao Trabalho

\begin{tabular}{|c|c|c|c|c|c|c|c|}
\hline Modelos & $N$ & $d f$ & $\chi^{2}$ & $\chi^{2} / d f$ & $G F I$ & CFI & $R M S E A(I C)$ \\
\hline \multicolumn{8}{|l|}{ Tema Clima ${ }^{1}$ : } \\
\hline $\begin{array}{l}8 \text { factores não- } \\
\text {-correlacionados }\end{array}$ & 567 & 1029 & 3663.21 & 3.56 & .76 & .66 & $.067(.065 ; .070)$ \\
\hline $\begin{array}{l}8 \text { factores não- } \\
\text {-correlacionados, } \\
1 \text { factor de segunda } \\
\text { ordem }\end{array}$ & 567 & 1021 & 2619.45 & 2.57 & .82 & .80 & $.053(.050 ; .055)$ \\
\hline 8 factores correlacionados & 567 & 1003 & 2366.85 & 2.36 & .85 & .83 & $.049(.046 ; .052)$ \\
\hline \multicolumn{8}{|l|}{ Tema Estrutura $^{2}$ : } \\
\hline $\begin{array}{l}2 \text { factores não- } \\
\text {-correlacionados }\end{array}$ & 567 & 62 & 436.14 & 7.03 & .91 & .75 & $.103(.098 ; .116)$ \\
\hline $\begin{array}{l}2 \text { factores não- } \\
\text {-correlacionados, } \\
1 \text { factor de segunda } \\
\text { ordem }\end{array}$ & 567 & 61 & 259.52 & 4.25 & .93 & .87 & $.08(.066 ; .085)$ \\
\hline 2 factores correlacionados & 567 & 61 & 259.52 & 4.25 & .93 & .87 & $.08(.066 ; .085)$ \\
\hline \multicolumn{8}{|l|}{$\begin{array}{l}\text { Tema Atitudes face ao } \\
\text { Trabalho }{ }^{3}:\end{array}$} \\
\hline $\begin{array}{l}2 \text { factores não- } \\
\text {-correlacionados }\end{array}$ & 567 & 247 & 1107.77 & 4.49 & .86 & .84 & $.08(.074 ; .083)$ \\
\hline $\begin{array}{l}2 \text { factores não- } \\
\text {-correlacionados, } \\
1 \text { factor de segunda } \\
\text { ordem }\end{array}$ & 567 & 246 & 765.21 & 3.11 & .89 & .90 & $.06(.056 ; .066)$ \\
\hline 2 factores correlacionados & 567 & 246 & 765.21 & 3.11 & .89 & .90 & $.06(.056 ; .066)$ \\
\hline \multicolumn{8}{|c|}{$\begin{array}{l}{ }^{1} \text { Em todos os modelos os pesos dos itens nos factores latentes são moderados a elevados } \\
\quad(\text { mínimo } \lambda=.32 ; \text { máximo } \lambda=.84) \text { e fortemente significativos }(p<.001) ;\end{array}$} \\
\hline \multicolumn{8}{|c|}{$\begin{array}{l}2 \text { Em todos os modelos os pesos dos itens nos factores latentes são moderados a elevados } \\
\text { (mínimo } \lambda=.28 ; \text { máximo } \lambda=.75) \text { e fortemente significativos }(p<.001) ;\end{array}$} \\
\hline $\begin{array}{c}3 \text { Em todos os modelos os } p \\
\quad \text { (mínimo } \lambda=.30 ; \text { máximo } \lambda\end{array}$ & . & $t \in$ & s fact & 1 & es & $m$ & rados a elevados \\
\hline
\end{tabular}

Tal como podemos observar relativamente ao tema Clima, dos três modelos testados o que revela os melhores índices de adequação é o modelo a oito factores correlacionados. De facto, a análise dos índices de adequação 
absolutos (GFI, CFI e RMSEA), bem como o índice qui-quadrado relativo mostra que este modelo atinge valores aceitáveis, $\mathrm{GFI}=.85 ; \mathrm{CFI}=.83$; RMSEA $=.049(\mathrm{IC}=.046$ a .052). Neste modelo, os pesos estandardizados dos itens nos respectivos factores latentes apresentaram-se, de um modo geral, moderados a elevados (de $\lambda=.32$ a $\lambda=.84$ ), e as correlações entre factores mostraram-se moderadas a fortes (de $r=-.12$ a $r=.90)$. No entanto, as correlações entre as dimensões realização pessoal e sobrecarga de papéis e entre cooperação e sobrecarga de papéis não se revelaram significativas. De uma forma geral, este modelo confirmatório segue, então, a proposta original de Glisson e Hemmelgarn (1998).

Nos restantes modelos, os índices de adequação são mais baixos e, no caso do modelo não correlacionado, os valores dos índices de adequação absolutos mostram-se abaixo dos valores normais referenciados na literatura (e.g., $\mathrm{GFI}=.75 ; \mathrm{CFI}=.65)$. Os resultados apresentados no Quadro 3 mostram ainda que as associações fortes obtidas entre as diferentes dimensões do tema Clima sugerem a sua agregação e coerência em torno deste mesmo tema.

Note-se que em todos os modelos, os índices de adequação aumentaram consideravelmente os seus valores quando retiramos da dimensão sobrecarga de papéis o item "Com que frequência tem de trabalhar horas extraordinárias?", e da dimensão crescimento e desenvolvimento o item "Esta organização enfatiza o crescimento e o desenvolvimento", uma vez que possuíam estimativas de erro de medida associadas bastante elevadas e, por este facto, perturbavam os modelos de medida. Neste sentido, o tema Clima mantém oito das nove dimensões originais, mas perde dois itens na nossa análise de validação de construto.

A análise da consistência interna de cada uma destas dimensões revelou que os alfas de Cronbach que obtivemos nesta amostra são, genericamente, mais baixos do que aqueles relatados pelos autores da escala original, ainda que aceitáveis. Assim, as dimensões despersonalização, crescimento e desenvolvimento e cooperação possuem um nível de consistência interna moderado $^{5}$ (respectivamente, $\alpha=.61 ; \alpha=.68 ; \alpha=.68$ ), enquanto as dimensões desenvolvimento pessoal, sobrecarga de papéis, clareza de papéis e conflito de papéis possuem um bom nível de consistência interna (respectivamente, $\alpha=.73 ; \alpha=.74 ; \alpha=.77 ; \alpha=.78)$. A dimensão exaustão emocional atingiu um nível de consistência interna bastante elevado $(\alpha=.83)$.

No que toca ao tema Estrutura, e como podemos verificar no Quadro 1, os dois modelos que mostram uma adequação mais razoável são o modelo a dois factores correlacionados e o modelo a dois factores com um

5 Os níveis de consistência interna apresentados foram classificados como moderados, elevados ou bastante elevados tendo por base a meta-análise realizada por Peterson (1994) a diversas publicações internacionais que apresentavam indicadores de alfa de Cronbach. 
factor de segunda ordem. Na realidade, estes dois modelos possuem parâmetros e indicadores de adequação idênticos. Isto acontece porque os constrangimentos impostos no modelo de segunda ordem para atingir a sua identificação fazem equivaler os graus de liberdade neste e no modelo a dois factores correlacionados. Contudo, a interpretação de um e outro modelo é substancialmente diferente. De forma a mantermos a coerência com o modelo original proposto por Glisson e Hemmelgarn (1998), optámos por seleccionar o modelo a dois factores correlacionados e utilizá-lo nas análises subsequentes.

Em ambos os modelos anteriores verificámos que ao retirar um item da escala de formalização (ou seja, o item "A utilização dos procedimentos estabelecidos é constantemente enfatizada") e outro item da escala de hierarquia de autoridade (o item "A forma como o trabalho é feito depende, essencialmente, da pessoa que o faz"), os índices de adequação melhoravam substancialmente.

Como se pode observar, também, no Quadro 1, o modelo a dois factores não correlacionados não atinge os índices de adequação considerados aceitáveis e é, por isso, preterido relativamente aos restantes modelos.

A análise da consistência interna das dimensões formalização e hierarquia de autoridade mostra valores aceitáveis (respectivamente, $\alpha=.69$; $\alpha=.70)$.

Por fim, e no que diz respeito ao tema Atitudes face ao Trabalho, os resultados apresentados no Quadro 1 mostram que o modelo que apresentou os piores níveis de adequação foi o modelo a dois factores não correlacionados. Tal como para o tema anterior, o modelo a dois factores relacionados e o modelo com dois factores e um factor de segunda ordem mostram-se razoavelmente adequados e com indicadores de qualidade aceitáveis. Tal como fizemos referência no tema anterior, estes dois modelos são idênticos em termos dos seus graus de liberdade e restantes indicadores estatísticos devido aos constrangimentos impostos no modelo de segunda ordem. Para este tema, vamos seguir a opção que tomámos no tema Estrutura, aceitando o modelo a dois factores correlacionados.

No que diz respeito à análise da consistência interna das dimensões satisfação com o trabalho e implicação organizacional, os valores de alfa de Cronbach encontrados mostraram-se elevados (respectivamente, $\alpha=.86$; $\alpha=.83$ ).

Análise à sensibilidade do questionário de clima: Os temas Clima, Estrutura, e Atitudes face ao Trabalho

Uma vez estabilizada a estrutura factorial do questionário, apresentamos, no Quadro 2 as pontuações médias obtidas pelos inquiridos nas diferentes dimensões do questionário de clima organizacional, bem como as respec- 
tivas medidas de assimetria e curtose e outras medidas descritivas. De uma forma global, podemos verificar que as dimensões validadas pela análise factorial confirmatória seguem uma distribuição aproximadamente normal, como pode ser testemunhado pelos valores de assimetria e curtose e, em especial, pelos rácios destas duas medidas pelos respectivos erros padrão. Exceptuam-se as dimensões realização pessoal e implicação. Estas duas dimensões mostram desvios à normalidade. No caso da dimensão realização pessoal observamos uma distribuição enviesada à direita, ou seja, com os participantes a responderem nos pontos mais elevados da escala, com um aspecto leptocurtico, isto é, com uma elevada concentração de frequências nestes mesmos pontos. Por outro lado, a dimensão implicação mostra, igualmente, uma distribuição enviesada à direita, mas desta feita com um aspecto platicurtico, ou seja, com uma maior dispersão de frequências pelos diferentes pontos da escala.

Quadro 2. Medidas descritivas das dimensões do Questionário de Clima Organizacional (total da amostra)

\begin{tabular}{|c|c|c|c|c|c|c|c|c|c|c|c|c|}
\hline $\begin{array}{l}\text { Medidas descri- } \\
\text { tivas }\end{array}$ & 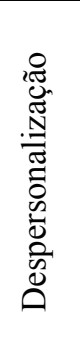 & 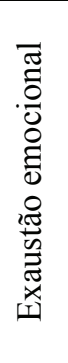 & 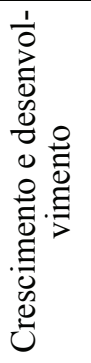 & 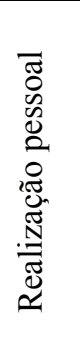 & 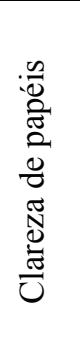 & 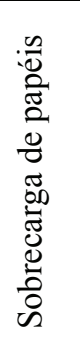 & 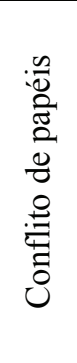 & 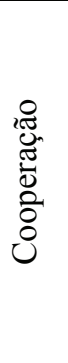 & 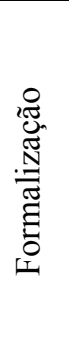 & 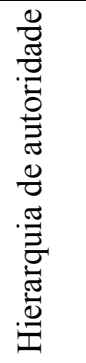 & 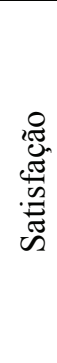 & 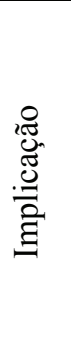 \\
\hline Média & 2.02 & 2.62 & 2.49 & 3.73 & 3.52 & 3.07 & 2.64 & 2.98 & 3.15 & 3.14 & 3.38 & 3.55 \\
\hline Desvio-padrão & .59 & .65 & .74 & .52 & .66 & .58 & .55 & .49 & .55 & .50 & .57 & .58 \\
\hline Mínimo & 1.00 & 1.00 & 1.00 & 1.50 & 1.33 & 1.29 & 1.00 & 1.50 & 1.29 & 1.33 & 1.64 & 1.77 \\
\hline Máximo & 3.60 & 4.50 & 5.00 & 5.00 & 5.00 & 4.71 & 4.22 & 4.67 & 5.00 & 4.60 & 4.91 & 5.00 \\
\hline Simetria & .26 & .15 & .28 & -.70 & -.23 & -.001 & -.10 & .10 & .03 & -.004 & -.10 & -.32 \\
\hline $\begin{array}{l}\text { Racio Simetria } \\
\text { / Erro padrão }\end{array}$ & 2.80 & 1.61 & 2.93 & -7.44 & -2.44 & -.01 & -1.10 & 1.01 & .32 & -.04 & 1.04 & -3.37 \\
\hline Curtose & -.53 & -.18 & -.07 & 1.38 & .07 & -.23 & -.03 & .06 & .40 & .14 & .08 & -.37 \\
\hline $\begin{array}{l}\text { Racio Curtose / } \\
\text { Erro padrão }\end{array}$ & -2.84 & -.95 & -.39 & 7.37 & .37 & -1.22 & -.17 & .34 & 2.13 & .73 & .40 & -1.96 \\
\hline
\end{tabular}

Para além disto, e a partir de uma análise de medidas repetidas, tomando os factores encontrados como variáveis intra-sujeitos, evidencia-se 
que os inquiridos pontuam, genericamente, de forma diferente nas diversas dimensões do questionários, $F(11,7480)=531.22, p<.001, \eta_{\mathrm{p}}{ }^{2}=.44$. No seguimento desta análise, realizámos testes $t$ contra o ponto médio (valor 3 ) da escala utilizada $(1=$ nunca a $5=$ sempre $)$, de modo a verificar como é que as respostas dos inquiridos se posicionam nas diferentes dimensões do questionário, bem como retirar algumas conclusões globais acerca do clima percepcionado, das percepções relativas à estrutura organizacional e da satisfação e implicação expressas pelos inquiridos.

Desta forma, na dimensão despersonalização a média de respostas dos inquiridos $(M=2.02, D P=.59)$ encontra-se abaixo do ponto médio da escala, $t(680)=-43.17, p<.001$, verificando-se o mesmo quando avaliam a exaustão emocional $(M=2.62, D P=.65), t(680)=-15.18, p<.001$, e a dimensão crescimento e desenvolvimento, $(M=2.49, D P=.74), t(680)=$ $-17.79, p<.001$. Na dimensão realização pessoal, as respostas dos inquiridos situam-se significativamente acima do ponto médio da escala $(M=3.73$, $D P=.52), t(680)=37.06, p<.001$, bem como na dimensão clareza de papéis, $(M=3.52, D P=.66), t(680)=20.28, p<.001$, e na dimensão sobrecarga de papéis $(M=3.07, D P=.58), t(680)=3.22, p<.001$. A dimensão conflito de papéis é avaliada abaixo do ponto médio pelos inquiridos $(M=$ $2.64, D P=.55), t(680)=-17.07, p<.001$. A dimensão cooperação foi avaliada no ponto médio da escala $(M=2.98, D P=.49), t(680)=-1.19, p<.24$.

Analisando agora as respostas médias dos inquiridos no tema Estrutu$r a$, e mais especificamente nas dimensões formalização e hierarquia de autoridade, as suas respostas situam-se acima do ponto médio da escala ( $M$ $=3.15, D P=.55), t(680)=6.99, p<.001$ e $(M=3.14, D P=.50), t(680)=$ 7.71, $p<.001$, respectivamente.

Finalmente, e relativamente ao tema Atitudes face ao Trabalho, os inquiridos respondem acima do ponto médio da escala quer na dimensão satisfação com o trabalho $(M=3.88, D P=.57), t(680)=17.29, p<.001$, quer na dimensão implicação à organização $(M=3.55, D P=.58), t(680)=$ 24.78, $p<.001$.

De uma forma geral, estes resultados mostram que as escalas possuem sensibilidade para detectar diferentes tendências de resposta dos inquiridos em diferentes domínios do clima e estrutura organizacional, bem como das suas atitudes face ao trabalho.

Para analisar, de uma forma ainda mais específica, a sensibilidade do questionário de clima relativamente a inquiridos classificados de acordo com diferentes variáveis sociodemográficas, realizámos diferentes análises de variância, com comparações post-hoc, analisando diferenças nas respostas dos participantes às dimensões deste instrumento em função da ocupação de 
um cargo de chefia, das funções exercidas, do sexo e da idade 6 . Apresentamos, de seguida, apenas as comparações post-hoc que se revelaram significativas (teste de Tukey, $p<.05$ ). Note-se que a dimensão realização pessoal não mostrou sensibilidade relativamente a nenhuma destas variáveis de caracterização sociodemográfica.

\section{Tema - Clima}

Despersonalização. A análise de sensibilidade efectuada sobre esta dimensão mostrou que esta diferencia bem indivíduos do sexo masculino e feminino, $F(1,597)=4.18, p<.04, \eta_{\mathrm{p}}{ }^{2}=.01$, e indivíduos que ocupam diferentes funções, $F(8,590)=3.35, p<.001, \eta_{\mathrm{p}}{ }^{2}=.004$. Assim, os inquiridos do sexo feminino mostraram níveis de despersonalização mais baixos $(M=$ $1.98)$ do que os inquiridos do sexo masculino $(M=2.11)$. Adicionalmente, profissionais de serviço social $(M=2.19)$ e formadores/professores $(M=$ 2.18) apresentam níveis de despersonalização superiores quando comparados com educadores $(M=1.84)$ e psicólogos $(M=1.82)$.

Exaustão emocional. A análise da sensibilidade desta dimensão mostrou que esta diferencia bem os inquiridos em função da idade, $F(3,591)=$ 3.18, $p<.02, \eta_{\mathrm{p}}{ }^{2}=.02$, da função que realizam $F(8,590)=3.29, p<.001$, $\eta_{\mathrm{p}}{ }^{2}=.04$, e do exercício de cargos de chefia, $F(1,594)=4.30, p<.04, \eta_{\mathrm{p}}{ }^{2}=$ .01 . Assim, verifica-se que os inquiridos mais jovens (isto é, até aos 29 anos) são os que manifestam níveis de exaustão emocional inferiores $(M=2.52)$. Estes valores diferem significativamente dos inquiridos com idades compreendidas entre os 30 e os 35 anos $(M=2.72), F(3,659)=2.86, p<.04, \eta_{\mathrm{p}}{ }^{2}$ $=.03$. Já no que diz respeito à função exercida podemos verificar que esta dimensão diferencia bem os psicólogos $(M=2.43)$ dos educadores $(M=$ 2.43), que reportam níveis mais baixos de exaustão emocional, e os coordenadores $(M=2.80)$ dos professores/formadores $(M=2.81)$, que apresentam níveis de exaustão mais elevados. Finalmente, verificou-se, também, que esta escala diferencia bem os inquiridos que ocupam lugares de chefia ( $M=$ 2.75) dos que não ocupam estes lugares $(M=2.59)$.

Crescimento e desenvolvimento. A análise de sensibilidade a esta dimensão mostrou que esta diferencia bem os indivíduos que ocupam diferentes funções, $F(8,590)=4.44, p<.001, \eta_{\mathrm{p}}{ }^{2}=.06$. Desta forma, inquiridos com funções menos especializadas, como os auxiliares $(M=2.77)$ apresentam valores mais elevados nesta dimensão do que inquiridos com funções mais especializadas, como por exemplo os profissionais de serviço social ( $M$ $=2.20)$ e os profissionais de saúde $(M=2.33)$.

${ }^{6}$ A variável idade foi recodificada de uma variável contínua para outra com escalões definidos, determinados através da utilização da partição quartílica. 
Clareza de papéis. Nesta dimensão verificaram-se diferenças entre inquiridos que ocupam diferentes funções, $F(8,590)=3.14, p<.002, \eta_{\mathrm{p}}{ }^{2}=$ .04 , e entre inquiridos que ocupam ou não lugares de chefia, $F(1,594)=$ $3.79, p<.05, \eta_{\mathrm{p}}{ }^{2}=.01$. Observamos que existe maior percepção de clareza de papéis junto dos auxiliares $(M=3.69)$ quando comparados com os assistentes sociais $(M=3.28)$ e junto daqueles que ocupam um lugar de chefia $(M$ $=3.65)$, em contraste com as não chefias $(M=3.50)$.

Conflito de papéis. Esta dimensão permite diferenciar inquiridos em diferentes escalões etários, $F(3,591)=4.78, p<.003, \eta_{\mathrm{p}}{ }^{2}=.002$, que ocupam diferentes funções, $F(8,590)=5.23, p<.001, \eta_{\mathrm{p}}{ }^{2}=.07$, bem como inquiridos do sexo feminino e masculino, $F(1,597)=3.95, p<.05, \eta_{\mathrm{p}}{ }^{2}=$ .01. Assim, verificámos que são os inquiridos com idades compreendidas entre os 30 e os 35 anos que percebem maior conflito de papéis $(M=2.76)$, pelo menos quando comparados com os inquiridos com idades entre os $16 \mathrm{e}$ os 19 anos $(M=2.61)$ e com mais de 46 anos $(M=2.57), F(3,659)=3.94$, $p<.001, \eta_{\mathrm{p}}{ }^{2}=.02$. Já no que diz respeito à diferenciação de inquiridos que desempenham diferentes funções, observámos que são aqueles que ocupam funções de coordenação, os formadores / professores e os profissionais de serviço social que percebem maior conflito de papéis (respectivamente, $M=2.82, M=2.79, M=2.83$ ), pelo menos quando comparados com os inquiridos que exercem funções de auxiliares $(M=2.45)$. Por fim, detectamos, ainda, uma diferenciação entre inquiridos do sexo feminino e do sexo masculino no que diz respeito a esta dimensão. Assim, são as inquiridas que mostram maior conflito de papéis $(M=3.16)$ quando comparadas com os inquiridos $(M=3.04)$.

Sobrecarga de papéis. Verificámos que esta dimensão mostra sensibilidade na diferenciação de inquiridos com idades diferentes, $F(3,591)=$ 7.54, $p<.001, \eta_{\mathrm{p}}{ }^{2}=.04$, com ocupação ou não de um lugar de chefia, $F(1$, $594)=9.09, p<.003, \eta_{\mathrm{p}}{ }^{2}=.02$, e com diferentes funções, $F(8,590)=10.91$, $p<.001, \eta_{\mathrm{p}}{ }^{2}=.13$. Deste modo, são os inquiridos mais velhos (isto é, com idades superiores a 46 anos) que percebem menor sobrecarga de papéis ( $M$ $=2.93$ ), pelo menos quando comparados com os inquiridos com idades compreendidas entre os 30 e os 35 anos $(M=3.21)$. Por outro lado, os inquiridos com funções de chefia, são aqueles que manifestam mais sobrecarga de papéis $(M=3.26$; inquiridos que não ocupam cargos de chefia: $M=$ 3.06). Por fim, os inquiridos com funções administrativas e auxiliares percebem níveis inferiores de sobrecarga de papéis (respectivamente, $M=2.85$; $M=2.80$ ), pelo menos quando comparados com os inquiridos com funções mais especializadas, como os profissionais de serviço social $(M=3.44)$.

Cooperação. Nesta dimensão apenas se verificaram diferenças nos inquiridos que ocupam, ou não, um lugar de chefia. Desta forma, aqueles que não ocupam uma posição de chefia pontuam mais alto nesta sub-escala 
$(M=3.00)$ do que aqueles que ocupam $(M=2.87), F(1,665)=9.74$, $p<.001, \eta_{\mathrm{p}}^{2}=.01$.

\section{Tema - Estrutura}

Formalização. Esta dimensão mostrou-se sensível a diferenças ao nível da idade dos inquiridos, $F(3,591)=6.96, p<.001, \eta_{\mathrm{p}}{ }^{2}=.03$, e da função que realizam, $F(3,590)=6.44, p<.001, \eta_{\mathrm{p}}{ }^{2}=.08$. Assim, são os inquiridos mais velhos, ou seja, com idades superiores a 46 anos, que percebem maior formalização nas suas instituições $(M=3.27)$, ao contrário dos inquiridos com idades compreendidas entre os 16 e os 29 anos $(M=3.03)$ e entre os 30 e os 35 anos $(M=3.05)$. No que diz respeito à função exercida pelos inquiridos, constatámos que profissionais que exercem funções administrativas ou auxiliares (respectivamente, $M=3.33 ; M=3.33$ ), expressam maior percepção de formalização do que os restantes profissionais, nomeadamente os psicólogos $(M=2.81)$.

Hierarquia de autoridade. Esta dimensão mostrou-se sensível a diferenças no que diz respeito ao sexo dos inquiridos, $F(1,597)=4.30, p<.04$, $\eta_{\mathrm{p}}{ }^{2}=.01$, ao facto de ocuparem, ou não, um lugar de chefia, $F(1,594)=$ 4.57, $p<.03, \eta_{\mathrm{p}}{ }^{2}=.01$, e à função que desempenham presentemente, $F(8$, $590)=4.69, p<.001, \eta_{\mathrm{p}}{ }^{2}=.06$. Relativamente ao sexo, podemos constatar que são os inquiridos do sexo feminino que percebem maior hierarquia de autoridade na sua instituição $(M=3.16$; sexo masculino: $M=3.04)$. Por outro lado, são os inquiridos com cargos de chefia que percebem uma menor hierarquia de autoridade $(M=3.53)$, ao contrário dos que não exercem estes cargos $(M=3.68)$. Finalmente, os profissionais de saúde $(M=2.91)$ percebem menor hierarquia de autoridade nas suas organizações quando comparados com os auxiliares $(\mathrm{M}=3.37)$.

\section{Tema-Atitudes face ao Trabalho}

Satisfação com o trabalho. Esta dimensão apenas se mostrou sensível às diferentes funções exercidas pelos inquiridos, $F(8,621)=4.05, p<.001$, $\eta_{\mathrm{p}}{ }^{2}=.06$. Assim, auxiliares $(M=3.56)$ e educadores $(M=3.51)$ mostram-se mais satisfeitos com o seu trabalho, pelo menos quando comparados com os profissionais de saúde $(M=3.48)$ e os profissionais de serviço social $(M=$ 3.11).

Implicação organizacional. Quando analisamos esta dimensão podemos verificar que ela se mostrou apenas sensível ao facto dos inquiridos ocuparem ou não um lugar de chefia, $F(1,594)=4.57, p<.03, \eta_{\mathrm{p}}{ }^{2}=.01$. Assim, aqueles que ocupam lugares de chefia encontram-se mais implicados com a sua organização $(M=3.68)$ do que os que não assumem este tipo de responsabilidade $(M=3.53)$. 
Validade relativa a um critério: Associações entre Clima, Estrutura e Atitudes face ao Trabalho

Para determinar a validade concorrente do questionário de Clima Organizacional, analisaram-se as associações entre as dimensões que compõem os diferentes temas, em especial as associações entre o tema Clima e o tema Estrutura, e aquele primeiro e o tema Atitudes face ao Trabalho. Para tal, construíram-se indicadores de acordo com os resultados obtidos nas diferentes análises factoriais confirmatórias, através do cálculo da média das respostas dos inquiridos nos diferentes itens que compõem as diferentes dimensões do questionário. Os resultados destas correlações são apresentados no Quadro 3, e podem ser tomados como indicadores, ainda que provisórios, da validade relativa a um critério (i.e., validade concorrente) do tema Clima relativamente a outros temas medidos por este questionário.

Quadro 3. Correlações entre as dimensões do tema "Clima" e as dimensões dos temas "Estrutura" e "Atitudes face ao Trabalho"

\begin{tabular}{lcccc}
\hline & \multicolumn{2}{c}{ Estrutura } & \multicolumn{2}{c}{ Atitudes face ao Trabalho } \\
\cline { 2 - 5 } Clima & Formalização & $\begin{array}{c}\text { Hierarquia de } \\
\text { autoridade }\end{array}$ & Satisfação & Implicação \\
\hline Despersonalização & $.11^{* * *}$ & $.10^{* *}$ & $-.33^{* * *}$ & $-.36^{* * *}$ \\
Exaustão emocional & $.14^{* * *}$ & $.15^{* * *}$ & $-.42^{* * *}$ & $-.39^{* * *}$ \\
Crescimento e desen- & -.05 & $-.08^{*}$ & $.49^{* * *}$ & $.54^{* * *}$ \\
volvimento & .05 & -.01 & $.55^{* * *}$ & $.39^{* * *}$ \\
Realização pessoal & .02 & -.07 & $.63^{* * *}$ & $.66^{* * *}$ \\
Clareza de papéis & .02 & $.13^{* * *}$ & $-.24^{* * *}$ & $-.25^{* * *}$ \\
Sobrecarga de papéis & .05 & $.19^{* * *}$ & $-.43^{* * *}$ & $-.51^{* * *}$ \\
Conflito de papéis & $.09^{* *}$ & $.14^{* * *}$ & -.06 & $-.14^{* * *}$ \\
Cooperação & $.16^{* * *}$ & & & \\
${ }^{*} p<.05 ;{ }^{* *} p<.01 ;{ }^{* * *} p<.001$ & & &
\end{tabular}

Como se pode verificar no quadro acima, as associações entre as medidas de Clima e as dimensões formalização e hierarquia de autoridade (do tema Estrutura) são fracas, ainda que significativas. Assim, quanto mais os participantes percebem que os procedimentos de trabalho existentes na sua organização são rígidos (i.e., quanto maior a formalização percebida), mais eles exprimem sentimentos de exaustão emocional e despersonaliza- 
ção. A percepção de rigidez de procedimentos de trabalho aparece também associada a uma maior cooperação. Da mesma forma, quando olhamos para as correlações entre as diferentes dimensões de Clima e a dimensão hierarquia e autoridade, podemos verificar que quanto mais os inquiridos percebem que a sua instituição possui uma linha hierárquica mais vincada, mais expressam sentimentos de exaustão emocional, despersonalização; e maiores as percepções de sobrecarga e conflito de papéis. Novamente, são também estes participantes os que alegam perceber maior cooperação.

Como se referiu, as associações entre Clima e as duas dimensões do tema Estrutura apresentam-se pouco expressivas do ponto de vista numérico. Neste sentido, estes resultados parecem sugerir que o clima percebido parece não estar particularmente relacionado com a estrutura organizacional, pelo menos no que à formalização e hierarquia diz respeito. Contudo, se colocarmos a atenção agora sobre as dimensões das Atitudes face ao Trabalho, o panorama de associações torna-se mais evidente. De facto, os inquiridos manifestam maior satisfação no trabalho e maior implicação organizacional quanto mais a organização proporciona oportunidades de crescimento $e$ desenvolvimento profissional e de recompensa pelo trabalho realizado; quanto mais claro o modo como os inquiridos percebem o seu papel na organização; e quanto mais eles se percebem pessoalmente realizados na sua função. Pelo contrário, níveis inferiores de satisfação estão associados a maiores sentimentos de exaustão emocional e de despersonalização e a maior sobrecarga e conflito de papéis desempenhados na instituição. Curiosamente, a cooperação apresenta correlações negativas com a implicação organizacional, ou seja, os inquiridos exprimem menor implicação quanto maior a cooperação que percebem existir na sua instituição. Finalmente, e de uma forma global, os resultados desta análise parecem sugerir que, ao contrário da relação incipiente entre Clima e Estrutura, as Atitudes face ao Trabalho, ou seja a implicação e a satisfação organizacional, relacionam-se fortemente com o Clima, testemunhando a existência de validade concorrente deste instrumento.

\section{Discussão}

Neste artigo analisámos as qualidades psicométricas de um instrumento especificamente concebido para ser aplicado em instituições sociais - o Questionário de Clima Organizacional (Glisson \& Hemmelgran, 1998). Este instrumento apresentou indicadores de validade de construto e de consistência interna adequados. De facto, e tal como demonstram os respectivos índices de adequação dos modelos obtidos nas análises factoriais confirmatórias quer o tema Clima (composto por oito factores correlacionados dos nove 
factores originais), quer o tema Estrutura (composto por dois factores correlacionados), quer ainda o tema Atitudes face ao Trabalho (composto por dois factores correlacionados) mostraram indicadores de razoável adequação. A consistência interna destas dimensões revelou-se adequada a boa ou elevada. De uma forma genérica, a estrutura factorial obtida nas diferentes dimensões segue aproximadamente a proposta original de Glisson e Hemmelgarn (1998). Note-se, contudo, que os temas Tecnologia e Justiça não mostraram, na presente amostra, qualidade métricas consideradas adequadas para serem mantidos na escala final que apresentamos neste artigo. Em estudos futuros será conveniente rever os itens que compõem estes temas, procurando adaptá-los e validá-los de modo a aproximarmo-nos da escala original proposta pelos autores.

Ainda no que diz respeito às propriedades psicométricas do Questionário de Clima Organizacional, testámos a sensibilidade das diferentes dimensões que o compõem relativamente à amostra global e a algumas variáveis de caracterização sociodemográfica dos trabalhadores de organizações sociais. Estas análises permitem-nos verificar o poder discriminativo das diferentes dimensões deste instrumento relativamente a diferentes tipos de inquiridos. Isto significa que o presente instrumento de Clima Organizacional permite obter avaliações mais finas e compreender como diferentes indivíduos percebem, de forma diferente, determinado aspecto importante da vida organizacional. Assim, os testes à sensibilidade mostraram que as dimensões que melhor permitem uma diferenciação de inquiridos com diferentes características sociodemográficas são as de exaustão emocional, conflito de papéis e sobrecarga de papéis do tema Clima, bem como a dimensão hierarquia e formalização do tema Estrutura. As restantes dimensões são menos diferenciadoras, ainda que se tenham verificado bons indicadores de sensibilidade relativamente à variável sexo dos inquiridos, ao facto de ocuparem, ou não, um lugar de chefia, e no que diz respeito à função que ocupam presentemente na organização.

Finalmente, testámos, ainda que de forma provisória, a validade relativa a um critério do tema Clima (na sua vertente de validade concorrente), utilizando como variáveis-critério os dois temas de Estrutura e Atitudes face ao Trabalho presentes neste instrumento. As correlações que obtivemos permitem-nos mostrar que as associações entre as diferentes dimensões do tema Clima e as dimensões formalização e hierarquia de autoridade do tema Estrutura se apresentaram pouco expressivas. Estes resultados não suportam a validade relativa a um critério do tema Clima relativamente ao tema Estrutura. Isto significa que, neste tipo de organizações, dificilmente podemos prever as opiniões dos inquiridos relativamente às dimensões formalização e hierarquia de autoridade a partir das suas pontuações nas diferentes dimensões do clima organizacional analisadas por este instrumento. 
Contudo, as associações entre o tema Clima e o tema Atitudes face ao Trabalho revelaram-se mais expressivas. Assim, é de notar a contribuição das dimensões crescimento e desenvolvimento, clareza de papéis e realização pessoal para uma maior satisfação e implicação na organização. Paralelamente, verificámos também que as dimensões exaustão emocional, despersonalização e conflito de papéis são as que contribuem mais para que os profissionais se sintam menos satisfeitos com o trabalho que desenvolvem presentemente na organização, bem como contribuem para a sua menor implicação organizacional, resultados estes que suportam estudos empíricos anteriormente publicados (cf., Carvalho, 2007).

Não podemos deixar de apontar algumas limitações aos dados que apresentamos anteriormente. Assim, para que possa ser generalizada, este tipo de investigação deverá ser replicada utilizando uma amostra representativa de organizações sociais que inclua uma maior diversidade de enquadramentos institucionais. Por outro lado, os resultados que apresentamos decorrem de percepções que os colaboradores têm da sua organização. Todavia, as limitações dos dados recolhidos por auto-relato são bem conhecidas (e.g., Podsakoff \& Organ, 1986). Investigação subsequente deverá incluir abordagens metodológicas adicionais, designadamente entrevistas e observações em contexto de trabalho que permitam completar os dados obtidos através do Questionário de Clima Organizacional.

Estudos futuros a realizar com este instrumento devem também procurar obter mais evidência empírica relativamente às suas qualidades psicométricas, nomeadamente no que diz respeito à sua validade relativamente a um critério, quer procurando novas medidas critérios, quer distanciando a sua mensuração do momento em que se recolhem os dados com o questionário de Clima Organizacional (permitindo, assim, a obtenção de indicadores de validade preditiva e assegurando uma menor contaminação entre medida e critérios). Adicionalmente, seria interessante, também, obter indícios de validade convergente deste instrumento relativamente a outros instrumentos de Clima organizacional.

Finalmente, salientamos que nem o contexto organizacional, nem um clima positivo, constituem por si só o objectivo a atingir pelas organizações sociais, mas sim um meio para promover o bem-estar dos seus utentes. De facto, a literatura mostra que o clima organizacional tem um impacto significativo na eficácia dos serviços prestados (e.g., Glisson \& Hemmelgarn, 1998). No entanto, este facto não explica, por si só, a ligação causal entre o clima organizacional e o serviço prestado. Neste sentido, investigadores, técnicos e decisores organizacionais deverão centrar os seus esforços em explorar empiricamente como é que o contexto organizacional, nomeadamente na sua vertente de clima organizacional, afecta a quantidade e a qualidade dos serviços prestados aos seus utentes. 


\section{Referências}

Altmann, R. (2000). Forecasting your organizational climate. Journal of Property Management, 65, 62-65.

Angle, H. L., \& Perry, J. L. (1986). An empirical assessment of organizational commitment and organizational effectiveness. Administrative Science Quarterly, 27, 1-14.

Arbuckle, J. L. (2006). Amos 7.0 user's guide. Chicago: SPSS.

Bentler, P. M. (1990). Comparative fit indexes in structural models. Psychological Bulletin, 107, 238-246.

Browne, M. W., \& Cudeck, R. (1989). Single sample cross-validation indices for covariance structures. Multivariate Behavioral Research, 24, 445-455.

Byrne, B. M. (2009). Structural equation modeling with AMOS: Basic concepts, applications, and programming (2nd ed.). New York: Routledge.

Calheiros, M., Moleiro, C., Garrido, M. V., Rodrigues, L., \& Martins, A. (2010). Questionário de clima organizacional (versão portuguesa). In M. Calheiros, P. Pereira, C. Moleiro, J. Graça, \& J. Patrício. Prevenção da institucionalização e apoio à família (PIAF). Relatório Final. CIS/ISCTE. Manuscrito não publicado.

Carvalho, I. (2007). Clima psicológico como preditor da saúde e do bem-estar de profissionais de saúde em contexto hospitalar. Psicologia, XXI, 27-58.

Cyphers, G. (2001). Report from the Child-Welfare Workforce Survey: State and county data and findings. Washington, DC: APHSA.

De Witte, K., \& De Cock, G. (1986). Organizational climate: It's relationship with managerial activities and communication structures. In G. Debus \& H. W. Schroiff (Eds). The psychology of work and organization: Current trends and issues (pp. 207-215). Amsterdam: North-Holland Publishing Company.

Festinger, L. (1954). A theory of social comparison processes. Human Relations, 7 , 117-140.

Forehand, G. A., \& Gilmer, B. H. (1964). Environmental variation in studies of organizational behavior. Psychological Bulletin, 62, 83-361.

Glisson, C. (2002). The organizational context of children's mental health services. Clinical Child and Family Psychology Review, 5, 233-253.

Glisson, C., \& Durick, M. (1988). Predictors of job satisfaction and organizational commitment in human service organizations. Administrative Science Quarterly, 33, 61-81.

Glisson, C., \& Hemmelgarn, A. L. (1998). The effects of organizational climate and interorganizational coordination on the quality and outcomes of children's service systems. Child Abuse \& Neglect, 22, 401-421.

Glisson, C., \& James, L. R. (2002). The cross-level effects of culture and climate in human service teams. Journal of Organizational Behavior, 23, 767-794.

Glisson, C., Dukes, D., \& Green, P. (2006). The effects of the ARC organizational intervention on caseworker turnover, climate, and culture in children's service systems. Child Abuse \& Neglect, 30, 855-880. 
Guion, R. (1973). A note on organizational climate. Organization Behavior and Human Performance, 9, 120-125.

Hellriegel, D., \& Slocum, J. (1974). Organizational climate: Measures, research, and contingencies. Academy of Management Journal, 17, 255-280.

Henggeler, S. W., \& Schoenwald, S. K. (1999). The role of quality assurance in achieving outcomes in MST programs. Journal of Juvenile Justice and Detention Services, 14, 1-17.

James, L. A., \& James, L. R. (1989). Integrating work environment perceptions: Explorations into the measurement of meaning. Journal of Applied Psychology, 74, 739-751.

James, L. J., \& Jones, A. P. (1974). Organizational climate: A review of theory and research. Psychological Bulletin, 81, 1096-1112.

James, L. R., \& Sells, S. B. (1981). Psychological climate: Theoretical perspectives and empirical research. In D. Magnusson (Ed.), Toward a psychology of situations: An interactional perspective (pp. 275-450). Hillsdale, New Jersey: Lawrence Erlbaum Associates.

James, L. R., James, L. A., \& Ashe, D. K. (1990). The meaning of organizations: The role of cognition and values. In B. Schneider (Ed.), Organizational climate and culture (pp. 40-84). San Francisco: Jossey-Bass.

Jones, A. P., \& James, L. R. (1979). Psychological climate: Dimensions and relationships of individual and aggregated work environment perceptions. Organizational Behavior \& Human Performance, 23, 201-250.

Jöreskog, K. G., \& Sorbom, D. (1984). LISREL VI user's guide. Mooresville, IN: Scientific Software.

Joyce, W. F., \& Slocum, J. W. (1982). Climate discrepancy: Refining the concepts of psychological and organizational climate. Human Relations, 35, 951-972.

Joyce, W. F., \& Slocum, J. W. (1984). Collective climate: Agreement as a basis for defining aggregate climates in organizations. Academy of Management Journal, 24, 721-742.

Kleinman, S., Handal, P, Enos, D., Searight, H., \& Ross, M. (1989). Relationship perceived between family climate and adolescent adjustment. Journal of Clinical Child Psychology, 18, 351-359.

Kottkamp, R., Mulhern, J., \& Hoy, W. (1987). Secondary school climate: A revision of the OCDQ. Educational Administrative Quarterly, 23, 31-48.

Locke, E. A. (1976). The nature and causes of job satisfaction. In M. D. Dunnette (Ed.), Handbook of industrial and organizational psychology (pp. 1297-1349). Chicago, IL: Rand McNally.

Miceli, M. P., \& Near, J. (1985). Characteristics of organizational climate and perceived wrong doing associates with whistle-blowing decisions. Personnel Psychology, 38, 525-544.

Mintzberg, H. (1979). The structuring of organizations: A synthesis of the research. Englewood Cliffs. NJ: Prentice Hall.

Moran, E. T., \& Volkwein, J. F. (1992). The cultural approach to the formation of organizational climate. Human Relations, 45, 19-47.

Mowday, R., Porter, L., \& Steers, R. (1982). Employee organization linkages. New York: Academic Press. 
Neves, J. (2001). Clima e cultura organizacional. In J. M. Carvalho Ferreira, J. Neves, \& A. Caetano (Orgs). Manual de psicossociologia das organizações (pp. 431-468). Lisboa: McGraw Hill.

Newman, J. E. (1977). Development of a measure of perceived work environment. Academy of Management Journal, 20, 520-534.

O’Driscoll, M. P., \& Evans, R. (1988). Organizational factors and perceptions of climate in three psychiatric units. Human Relations, 41, 371-388.

Ostroff, C., \& Schmitt, N. (1993). Configurations of organizational effectiveness and efficiency. Academy of Management Journal, 36, 1345-1361.

Paulin, M., Ferguson R. J., \& Bergeron, J. (2006), Service climate and organizational commitment: The importance of customer linkages. Journal of Business Research, 59, 906-915.

Payne, R., \& Pugh, D. (1975) Organization structure and organization climate. In M. D. Dunnette (Ed.), Handbook of industrial-organizational psychology (pp. 1125-1174). Chicago, IL: Rand McNally.

Peterson, R. A. (1994). A meta-analysis of Cronbach's coefficient alpha. Journal of Consumer Research, 21, 381-391.

Podsakoff, P. M., \& Organ, D. W. (1986). Self-reports in organizational research: Problems and prospects. Journal of Management, 12, 531-544.

Poole, M. S. (1985). Communication and organizational climates: Review, critique and a new perspective. In. R. D. McPhee \& P. K. Tompkins (Eds.), Organizational communication: Traditional themes and new directions (pp. 79-108). Newbury Park, CA: Sage.

Schneider, B. (1975). Organizational climates: An essay. Personnel Psychology, 28, 447-479.

Schneider, B., \& Reichers, A. E. (1983). On the etiology of climates. Personnel Psychology, 36, 19-39.

Shim, M. (2010). Factors influencing child welfare employee's turnover: Focusing on organizational culture and climate. Children and Youth Services Review, 32, 847-856.

Soloman, E. E. (1986). Private and public sector managers: An empirical investigation of job characteristics and organizational climate. Journal of Applied Psychology, 71, 247-259.

Turnipseed, D. (1980). Evaluation of healthcare work environments via a social climate scale: Results of a field study. Hospital Health Services Administration $35,245-261$.

Verbeke, W., Volgering, M., \& Hessels, M. (1998). Exploring the conceptual expansion within the field of organizational behaviour: Organizational climate and organizational culture. Journal of Management Studies, 35, 303-329.

Williams, L. J., \& Hazer, J. T., (1986). Antecedents and consequences of satisfaction and commitment in turnover models: A reanalysis using latent variable structural equation methods. Journal of Applied Psychology, 71, 219-231.

Witt, L. A. (1989). Person situation effects in the explanation of self-presentation on the job: Locus of control and psychological climate and job satisfaction. Journal of Social Behavior and Personality 4, 521-530.

Yoo, J., Brooks, D., \& Patti, R. (2007). Organizational constructs as predictors of effectiveness in child welfare interventions. Child Welfare, 86, 53-78. 
Anexo I: Dimensões e itens do questionário de Clima Organizacional para Organizações de Intervenção Social (versão portuguesa)

\section{Tema - Clima}

\section{Despersonalização}

Sinto que trato alguns utentes/clientes de forma impessoal

Tenho-me tornado mais insensível face aos outros desde que comecei a trabalhar aqui

Preocupa-me que este trabalho me esteja a tornar insensível

Por vezes apercebo-me que não me preocupo realmente com o que acontece a alguns dos meus utentes/clientes

É difícil sentir-me próximo(a) dos meus utentes/clientes

\section{Exaustão emocional}

Sinto-me emocionalmente esgotado(a) com o meu trabalho

Sinto-me exausto(a) no final do meu dia de trabalho

Sinto-me fatigado(a) quando me levanto de manhã e tenho de enfrentar mais um dia no trabalho

Sinto-me esgotado(a) devido ao meu trabalho

Sinto que me esforço demasiado no meu trabalho

Sinto que emocionalmente estou a chegar ao meu limite

\section{Crescimento e desenvolvimento}

Esta organização recompensa a experiência, a dedicação e o empenho

Em geral, há mais oportunidades para progredir nesta organização do que em outro tipo de empregos

As oportunidades de progresso na minha função são muito maiores do que a dos que estão noutras funções

Esta organização proporciona várias oportunidades de promoção se trabalharmos para tal

\section{Realização pessoal}

Lido de forma muito eficaz com os problemas dos meus utentes/clientes

Sinto que influencio positivamente a vida de outras pessoas através do meu trabalho

Consigo facilmente criar um ambiente descontraído com os meus utentes/clientes

Sinto-me bem-disposto(a) depois de trabalhar de perto com os meus utentes/clientes

Neste trabalho tenho realizado muitas coisas que valem a pena

No meu trabalho lido facilmente com os problemas emocionais dos outros

\section{Clareza de papéis}

Compreendo como o meu desempenho é avaliado

As responsabilidades do meu trabalho estão claramente definidas

Sei o que as pessoas nesta organização esperam de mim

É bem informado(a) sobre aquilo que precisa saber?

Com que frequência os objectivos e propósitos do seu cargo estão claramente definidos?

Com que frequência é possível obter informação precisa sobre as políticas e os procedimentos administrativos? 


\section{Conflito de papéis}

Com que frequência acaba por fazer coisas que deveriam ser feitas de forma diferente?

Com que frequência tem de "contornar" uma regra para conseguir realizar uma tarefa?

Com que frequência se sente incapaz de satisfazer pedidos incompatíveis dos seus supervisores?

Com que frequência o seu trabalho interfere com a sua vida familiar?

Os interesses dos utentes/clientes são muitas vezes substituídos por preocupações administrativas (e.g., burocracia)

As normas e regulamento dificultam frequentemente o trabalho

A quantidade de trabalho que tenho interfere com a qualidade com que o faço

No meu trabalho tenho que fazer tarefas que vão contra a minha perspectiva

Há inconsistências nas normas e regulamento que tenho de seguir

\section{Sobrecarga de papéis}

Com que frequência os seus colegas apresentam sinais de stresse?

Por mais que eu faça, há sempre mais a ser feito

A quantidade de tarefas que tenho para fazer impede-me de fazer um bom trabalho

Não há pessoas suficientes na organização para realizar o trabalho

Quando inicio uma tarefa, não me é dado tempo suficiente para a completar

Com que frequência está sob forte pressão no seu trabalho?

\section{Cooperação}

Com que frequência existe atrito entre os seus colegas de trabalho?(*)

Há um sentimento de cooperação entre os meus colegas de trabalho

Quando tenho uma tarefa difícil a fazer, as pessoas da organização ajudam-me

Com que frequência os seus colegas confiam uns nos outros?

\section{Tema - Estrutura}

\section{Formalização}

Aqui, as pessoas fazem o trabalho da mesma forma todos os dias

Só há uma forma de fazer o trabalho - fazer como a chefia quer

Habitualmente, as circunstâncias são as mesmas de dia para dia

No desenvolvimento de cada tarefa, têm que se seguir os mesmos passos

Os mesmos procedimentos devem ser seguidos na maioria das situações

Devemos sempre seguir procedimentos operacionais rígidos

Quando temos um problema, devemos dirigir-nos à mesma pessoa para o solucionar

\section{Hierarquia de Autoridade}

Poucas coisas podem ser feitas antes que um supervisor ou coordenador aprove a decisão

Aqui, as ordens têm origem nos níveis hierárquicos superiores

Sinto que, na maioria das áreas, sou "chefe" de mim próprio(a)(*)

Em quase tudo o que faço, tenho que perguntar primeiro ao supervisor ou coordenador

Pode-se tomar decisões próprias sem ter que confirmar com ninguém(*)

Todas as decisões que tomo têm que ser aprovadas pelo supervisor ou coordenador 


\section{Tema - Atitudes face ao Trabalho}

\section{Satisfação com o trabalho}

Com que frequência se sente satisfeito(a) com o prestígio que o seu trabalho tem na comunidade?

Com que frequência se sente satisfeito(a) com a sua capacidade de fazer as coisas da forma mais correcta?

Com que frequência se sente satisfeito(a) com a oportunidade que tem de fazer coisas pelos seus utentes/clientes?

Com que frequência se sente satisfeito(a) com a oportunidade que tem de fazer algo que exija aplicar as suas competências?

Com que frequência se sente satisfeito(a) com a forma como as políticas da organização são postas em prática?

Com que frequência se sente satisfeito(a) com as possibilidades de progredir na carreira?

Com que frequência se sente satisfeito(a) com a liberdade que tem de agir de acordo com o que pensa?

Com que frequência se sente satisfeito(a) com a oportunidade de experimentar abordagens suas no trabalho com os seus utentes/clientes?

Com que frequência se sente satisfeito(a) com as suas condições de trabalho?

Com que frequência se sente satisfeito(a) com o reconhecimento que recebe por fazer um bom trabalho?

Com que frequência se sente realizado(a) com o que obtém do seu trabalho?

\section{Implicação organizacional}

Sinto orgulho em dizer aos outros que faço parte desta organização

Preocupo-me realmente com o destino desta organização

Não há muito a ganhar em continuar nesta organização indefinidamente

Considero que decidir trabalhar nesta organização foi um erro da minha parte(*)

Considero que, de todas as organizações, esta é a melhor para se trabalhar

Considero que, nas minhas circunstâncias actuais, não seriam precisas muitas alterações para sair desta organização(*)

Esta organização incentiva o "meu melhor" em relação ao meu desempenho no trabalho.

Os meus valores e os da organização são muito semelhantes

Sinto pouca lealdade para com esta organização(*)

Considero difícil concordar com as políticas desta organização em questões importantes relativas aos seus funcionários $(*)$

Estou muito contente por ter escolhido trabalhar nesta organização

Estou disposto(a) a empenhar-me para que esta organização tenha sucesso

Digo aos meus amigos que esta é uma boa organização para se trabalhar

(*) Itens com escala invertida 